\title{
Using pulmonary artery acceleration time to evaluate pulmonary hemodynamic changes on preterm infants with respiratory distress syndrome
}

\author{
Liling Wang ${ }^{1,2}$, Fengjuan Zhang ${ }^{2}$, Jiahui $\mathrm{Li}^{2}$, Zhijie Liu ${ }^{2}$, Yan $\mathrm{Kou}^{2}$, Yanting Song ${ }^{2}$, Haiyan $\mathrm{Xu}^{2}$, \\ Haiyan Wang ${ }^{3}$, Yulin Wang ${ }^{1}$
}

${ }^{1}$ Department of Pediatric Cardiology, Shandong Provincial Hospital, Cheeloo College of Medicine, Shandong University, Jinan, China; ${ }^{2}$ Department of Pediatrics, The First Affiliated Hospital of Shandong First Medical University, Jinan, China; ${ }^{3}$ Department of Medical Ultrasound, The First Affiliated Hospital of Shandong First Medical University, Jinan, China

Contributions: (I) Conception and design: L Wang, H Wang; (II) Administrative support: Y Wang, H Xu; (III) Provision of study materials or patients: L Wang, F Zhang, J Li; (IV) Collection and assembly of data: Z Liu, Y Kou, Y Song; (V) Data analysis and interpretation: H Wang, L Wang; (VI) Manuscript writing: All authors; (VII) Final approval of manuscript: All authors.

Correspondence to: Yulin Wang, MD. Department of Pediatric Cardiology, Shandong Provincial Hospital, Cheeloo College of Medicine, Shandong University, Jinan 250021, China. Email: wangyulin2116@163.com; Haiyan Wang, MD. Department of Medical Ultrasound, The First Affiliated Hospital of Shandong First Medical University, Jinan 250014, China. Email: wanghaiyan96@126.com.

Background: Pulmonary artery acceleration time (PAAT) is a reliable and non-invasive method for assessing pulmonary hemodynamics. To date, few studies have used PAAT to assess preterm infants, especially those with respiratory distress syndrome (RDS). This study aimed to assess changes in PAAT among preterm infants with RDS undergoing pulmonary surfactant (PS) therapy or not, and determine its potential effects on the pulmonary vascular disease (PVD) outcomes of preterm infants with RDS in the late postnatal period.

Methods: The risk of RDS was reviewed in 62 preterm infants with a gestational age of 26-31 weeks. The infants receiving PS therapy were allocated to the PS group, and the others were allocated to the control group. PAAT, right ventricular ejection time (RVET), and other ultrasonic parameters at 3 different time points after birth were studied and compared.

Results: Infants in the PS group had a significantly lower PAAT $(52.7 \pm 5.9$ vs. $59.6 \pm 8.7 ; \mathrm{P}=0.001)$ and PAAT/RVET $(0.30 \pm 0.03$ vs. $0.33 \pm 0.03 ; \mathrm{P}=0.001)$ than those in the control group at 36 weeks postmenstrual age (PMA). No significant increases in PAAT/RVET were detected at 3 different times for the PS group $(\mathrm{P}=0.117)$, but both PAAT and PAAT/RVET increased significantly with time after birth in the control group $(\mathrm{P}<0.001)$.

Conclusions: Preterm infants with RDS might still have PVD in the late postnatal period and thus require long-term follow-up observation. PAAT appears to be a reliable non-invasive screening measure for evaluating pulmonary hemodynamics in preterm infants with RDS and late PVD.

Keywords: Respiratory distress syndrome (RDS); surfactant therapy; preterm infant; pulmonary artery acceleration time (PAAT); echocardiograph

Submitted Jun 18, 2021. Accepted for publication Aug 17, 2021.

doi: $10.21037 /$ tp-21-341

View this article at: https://dx.doi.org/10.21037/tp-21-341 


\section{Introduction}

Neonatal respiratory distress syndrome (RDS) is a serious disease that is caused by the structural immaturity of the lungs and pulmonary surfactant (PS) deficiency, which can result in underinflated alveoli, hypoxemia, and respiratory acidosis (1). The administration of PS plays an important role in the management of RDS (2-5). Management methods for RDS have evolved gradually over the years; however, the disease remains a significant respiratory problem for preterm infants in the early postnatal period (2). Additionally, a critical stage of normal lung development is between gestational age of 20 and 30 weeks, which increases the risk of chronic lung disease or bronchopulmonary dysplasia (BPD) in very preterm infants (6).

Preterm birth can also disrupt the maturational development of pulmonary circulation, resulting in increased oxygen demand and poor vascular reactivity, and this can eventually lead to pulmonary vascular disease (PVD), even pulmonary hypertension (PH) (7). Preterm infants may develop RDS, pulmonary arteriole constriction, and alveolar collapse. Such pathological changes can make PVD worse (8-11). Previous studies have suggested that early echocardiographic evidence of PVD in preterm infants contributes to BPD and late PH $(12,13)$. Thus, the echocardiographic prediction of which premature infants may develop significant PVD is an important and growing field. Such prediction could enable targeted therapy with oxygen, pulmonary vasodilators, or different respiratory support strategies to be implemented. However, pulmonary hemodynamic assessment in newborns poses unique problems due to the technical challenges and risk of complications (14). Traditional imaging techniques may be inadequate in this regard. For example, chest X-ray radiographs cannot evaluate pulmonary vascular lesions (15). The use of right cardiac catheterization to directly measure pulmonary arterial pressure (PAP) is the gold standard for evaluating pulmonary vascular lesions in adults and children; however, it is seldom used for neonates in clinical practice as it is an invasive procedure that carries a high risk of complications (16).

Additionally, most commonly used echocardiographic parameters [e.g., pulmonary artery systolic pressure estimated using the sum of the tricuspid regurgitation (TR) pressure and the right atrial pressure] cannot be used in neonates because echocardiography cannot obtain satisfactory TR Doppler signals in almost $40 \%$ of patients, such signals are only measurable in less than half of patients with BPD and PH (17-19). Further, other indirect signs reflecting $\mathrm{PH}$, such as septal flattening and an increased right/left ventricular diameter ratio, often only occur as a result of moderate or severe increases in the right ventricular afterload; thus, these signs are neither sensitive nor specific $(13,20)$. To assess the severity and outcomes of early pulmonary vascular lesions, other parameters that have higher sensitivity and specificity than those mentioned above are urgently needed.

In recent years, pulmonary artery acceleration time (PAAT) has been shown to sensitively detect $\mathrm{PH}$ in childhood (21). PAAT has been confirmed to provide a reliable estimate of invasive pulmonary hemodynamic measures in children and neonates. Multiple studies have shown that PAAT does not rely on the presence of TR or an anatomical defect, and that PAAT values are negatively correlated with right heart catheterization-derived PAP and indexed pulmonary vascular resistance. PAAT can be used in the vast majority of patients to assess PAP $(21,22)$. To date, few studies have used PAAT on preterm infants, especially those with RDS, but in fact, it is very important to have a deeper understanding of the hemodynamics changes in those infants, as well as the increased pulmonary vascular resistance and transition difficulties in premature infants caused by pulmonary diseases. Thus, we hypothesized that preterm infants with RDS who required PS treatment would have more severe clinical symptoms and more significant pulmonary hemodynamic changes, and that even with PS treatment, late respiratory disease would be more likely to develop. This study sought to compare PAAT differences between preterm infants with RDS who needed PS treatment and those who did not. We also attempted to find a reliable non-invasive screening measure for evaluating pulmonary hemodynamics in premature infants with RDS and late respiratory disease.

We present the following article in accordance with the STROBE reporting checklist (available at https://dx.doi. org/10.21037/tp-21-341).

\section{Methods}

\section{Study population}

The First Affiliated Hospital of Shandong First Medical University is a 3 tertiary neonatal intensive care unit (NICU) and regional critical neonatal treatment transfer center. Preterm infants who were consecutively admitted to the NICU from January to December 2019 were included in 
this prospective cohort study. To be eligible to participate in this study, patients had to meet the following inclusion criteria: (I) have a gestational age (GA) of 26-31 weeks; and (II) have required nasal continuous positive airway pressure (nCPAP) support immediately after birth. Patients were excluded from the study if they met any of the following exclusion criteria: (I) had congenital heart disease [other than patent ductus arteriosus (PDA) or patent foramen ovale], major congenital malformations, early onset sepsis, severe asphyxia or a need for inotropes, arrhythmia, pulmonary hemorrhage, or a large PDA requiring surgical ligation; (II) had required mechanical ventilation (MV) within 1 week after birth; and/or (III) had poor-quality ultrasonic images.

\section{Study design}

Under our NICU's guidelines for the management of RDS, all preterm infants at possible risk of RDS should be started on nasal continuous positive airway pressure (nCPAP; PEEP 6-8 $\mathrm{cmH}_{2} \mathrm{O}$ ) from birth. Further, surfactant (Curosurf, Chiesi Farmaceutici, Italy, $200 \mathrm{mg} / \mathrm{kg}$ ) should be administered (via traditional intubation) when the fraction of inhaled oxygen exceeds 0.30 in the first 2 hours after birth on nCPAP. Sixtytwo preterm infants participated in this study. Of these infants, 32 received early PS treatment (the PS group), and $30 \mathrm{did}$ not (the control group). Echocardiography was performed at 3 different time points. For the PS group, the 3 timepoints were before PS administration, which represented the baseline characterization (T0), at 24 hours after PS administration (T1), and at 36 weeks postmenstrual age (PMA; T2). For the control group, the 3 time points were within 2 hours of birth (T0), at 24 hours of age (T1), and at 36 weeks PMA (T2).

All procedures performed in this study involving human participants were in accordance with the Declaration of Helsinki (as revised in 2013). This study was approved by the Ethics Committee of the First Affiliated Hospital of Shandong First Medical University, and informed consent was obtained from the children's parents or legal guardians.

The echocardiography was performed by a single operator, who was blinded to infant status at the time of data interpretation, using the CX-50 Cardiovascular Ultrasound System (Philips Medical Systems, USA) with an S12-4 transducer to obtain images from the parasternal long axis, short axis, and apical 4-chamber view. All measurements were taken repeatedly over three consecutive cardiac cycles, and all images were saved to the hard disk of the ultrasonic instrument for offline analysis.

The parameters for representing the hemodynamics of the right ventricle and pulmonary artery included pulmonary artery systolic pressure (calculated from the sum of the TR pressure difference and right atrial pressure), pulmonary artery valve PAAT, and RVET. Heart rate (HR) and the status of the ductus arteriosus were also measured. PAAT and RVET measurements were obtained from the parasternal short axis view, by placing the sample volume at the pulmonary valve orifice. PAAT was defined as the time from the onset of right ventricle ejection and peak flow velocity. RVET was defined as the time interval measured from the onset to the end of systole in ms (see Figure 1).

\section{Statistical analysis}

The statistical analysis was performed using SPSS 25.0. The count data are represented by $\mathrm{n}(\%)$ and were analyzed by a chi-square test. The measurement data are expressed as mean \pm standard deviation or median (inter quartile range) depending on whether the data were normally distributed or not. Analyses of variance were used to compare the clinical observational indicators of the PS and control groups. A generalized linear mixed model (with Bonferroni adjustment) was used to compare the ultrasonic values between the PS and control groups at different observation time points. A $\mathrm{P}$ value $<0.05$ was considered significant.

\section{Results}

\section{Population details and clinical parameters}

Of the 62 preterm infants recruited at birth, 3 died before hospital discharge, leaving 59 infants with data for analysis at 36 weeks PMA. Over the study period, all the infants had a normal HR (120-160 bpm), and none required inotrope support. Compared to the control group, the PS group had a higher rate of cesarean section, CPAP days, BPD, and hospital stays, and a lower 5-minute Apgar score, arterial blood gas $\mathrm{pH}$ value, and arterial partial pressure of oxygen at 1 hour after birth. There were no significant differences between the PS group and the control group in terms of GA, birth weight, head circumference, height, sex ratio, antenatal corticosteroids, maternal age, admission body temperature, time of transfer to the NICU, severe intraventricular hemorrhage, late onset sepsis, necrotizing enterocolitis, and retinopathy of prematurity (see Figure 2 and Table 1). 


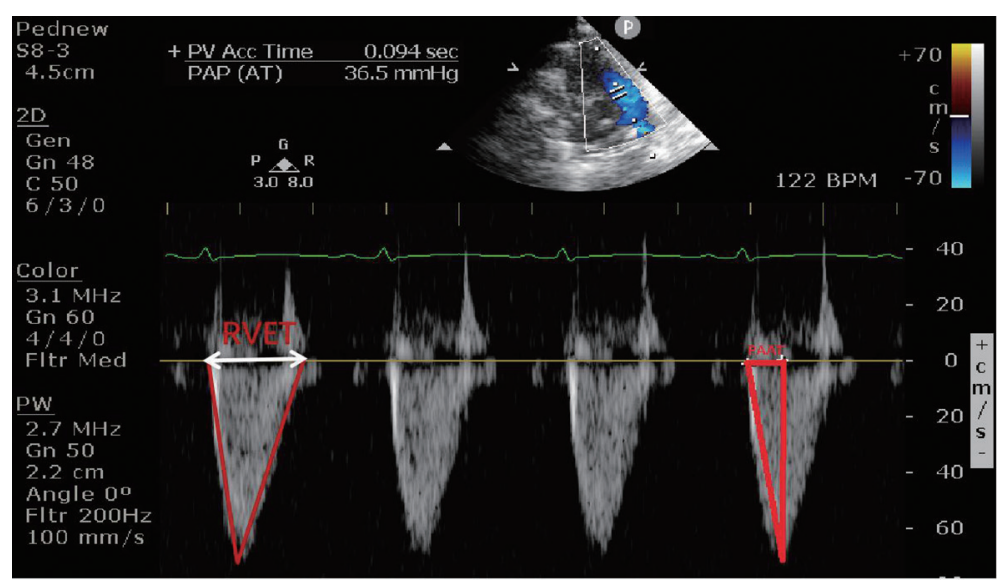

Figure 1 PAAT and RVET measurements. PAAT was measured at the center of the main pulmonary artery just at the pulmonary valve orifice in the parasternal short axis view in pulsed wave Doppler mode (the time interval between the onset of right ventricular ejection and peak flow velocity in ms). RVET was the time interval measured from the onset to the end of systole in ms. PAAT, pulmonary artery acceleration time; RVET, right ventricular ejection time.

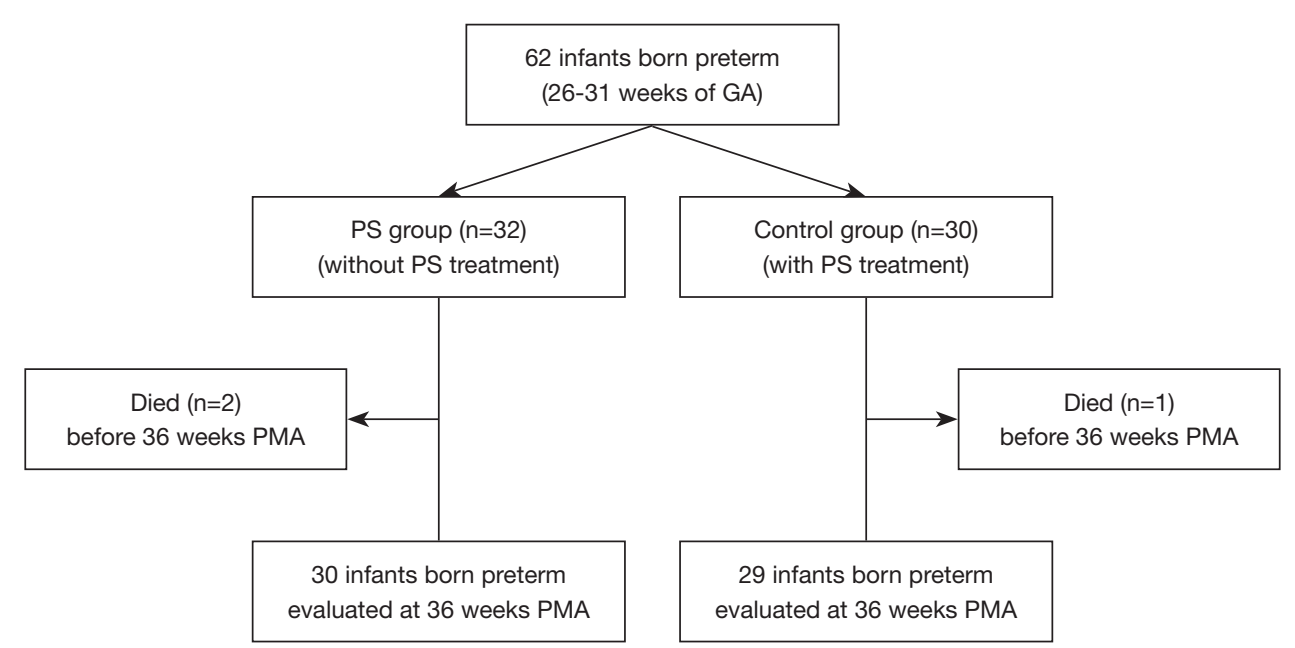

Figure 2 Population details. GA, gestational age; PMA, postmenstrual age.

\section{Comparison of the PS and control groups' respective ultrasonic parameters at the 3 time points (see Tables 2,3)}

\section{Baseline hemodynamics at T0}

There were no significant intergroup differences for PAAT $(45.7 \pm 7.0$ vs. $48.0 \pm 8.1 ; \mathrm{P}=0.231)$, or PAAT/RVET $(0.29 \pm 0.03$ vs. $0.28 \pm 0.04 ; \mathrm{P}=0.090)$. However, TR was observed in $56 \%$ of infants in the PS group, and $43.3 \%$ of infants in the control group $(\mathrm{P}=0.446)$. There were no significant differences between the two groups in terms of right ventricular systolic blood pressure $(18.9 \pm 3.7$ vs. $15.9 \pm$
$5.3 \mathrm{mmHg} ; \mathrm{P}=0.079)$.

\section{Comparison of the two groups in relation to} echocardiographic characterization at $\mathrm{T} 1$

At T1, infants who underwent PS treatment had a lower PAAT/RVET compared to the control group $(0.29 \pm 0.02 v s$. $0.31 \pm 0.03 ; \mathrm{P}=0.033)$, but no significant change was observed for PAAT. TR was measured, and right ventricular systolic blood pressure was estimated at $23.6 \pm 4.7 \mathrm{mmHg}$ in $25 \%$ of the infants in the PS group and $18.9 \pm 4.2 \mathrm{mmHg}$ in $20 \%$ in 
Table 1 Clinical parameters of preterm infants

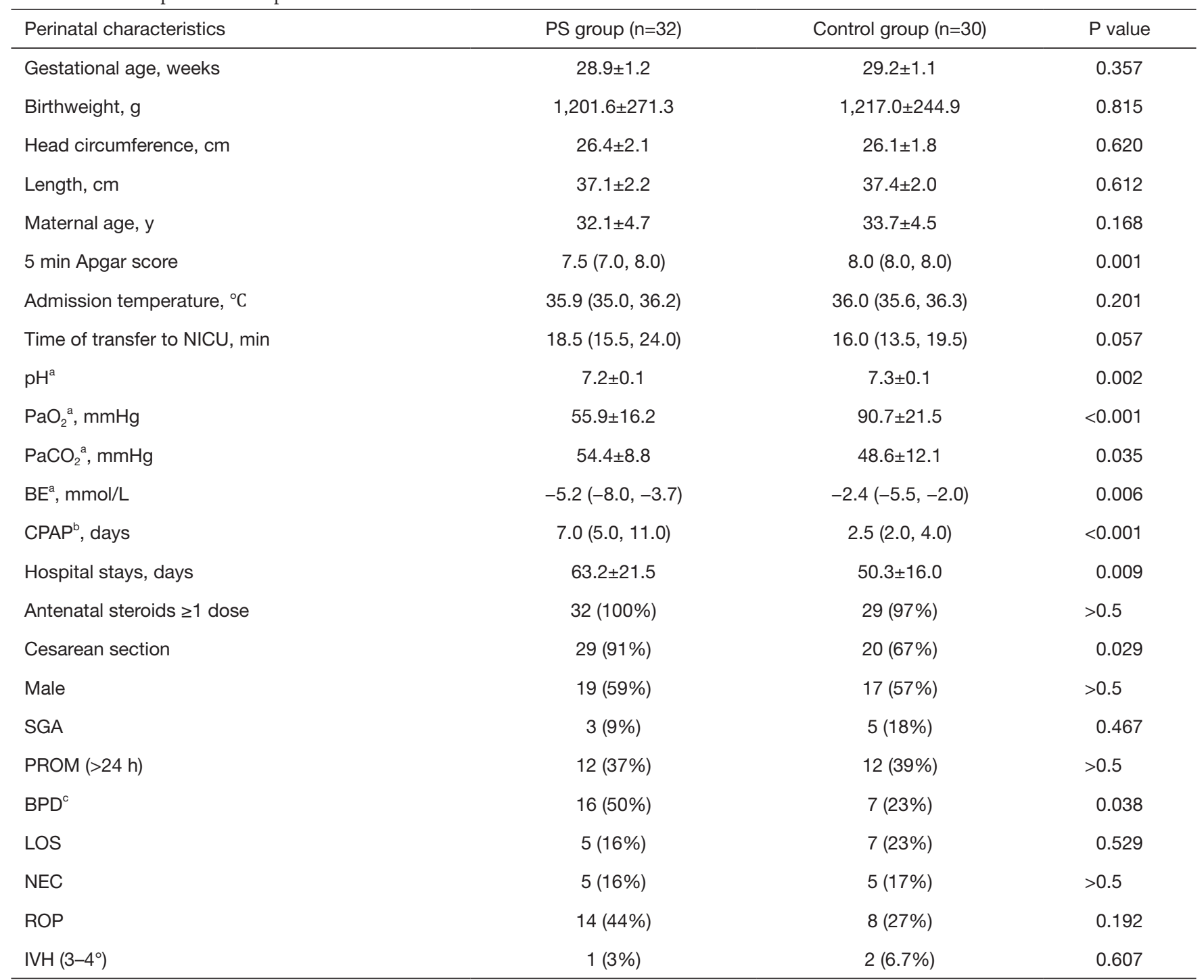

${ }^{a}$, arterial blood gas 1 hour after birth; ${ }^{b}$, the first time at which heated humidified high flow nasal cannula or air was changed; ${ }^{c}$, BPD as defined by the 2001 National Institute of Health BPD workshop (17). CPAP, continuous positive airway pressure; SGA, small for gestational age; PROM, prolonged rupture of membranes (>18 h); BPD, bronchopulmonary dysplasia; LOS, late onset sepsis; NEC, necrotizing enterocolitis; ROP, retinopathy of prematurity; IVH, intraventricular hemorrhage.

the control group, $\mathrm{P}=0.076$. No significant differences were found.

\section{Comparison of the two groups in relation echocardiographic characterization at T2}

At T2, infants in the PS group had a significantly lower PAAT $(52.7 \pm 5.9$ vs. $59.6 \pm 8.7 ; \mathrm{P}=0.001)$ and PAAT/RVET $(0.30 \pm 0.03$ vs. $0.33 \pm 0.03 ; \mathrm{P}=0.001)$ compared to the control group. TR and right ventricular systolic blood pressure were measured only in 2 patients in the PS group, and in 3 patients in the control group $(6.3 \%$ vs. $10.0 \% ; \mathrm{P}=0.667)$.

\section{Intergroup comparison of ultrasonic parameters at the 3 time points}

For the PS group, while differences in PAAT were found between $\mathrm{T} 0$ and $\mathrm{T} 1$, and $\mathrm{T} 0$ and $\mathrm{T} 2$, no significant increases in PAAT/RVET were detected at the three different time points $(\mathrm{P}=0.117)$. For the control group, both PAAT and PAAT/RVET increased significantly with time 
Table 2 The PAAT (ms) values of the two groups

\begin{tabular}{lccc}
\hline Time & PS group & Control group & $P$ \\
\hline T0 & $45.7 \pm 7.0^{\mathrm{A}}$ & $48.0 \pm 8.1^{\mathrm{A}}$ & 0.231 \\
T1 & $50.6 \pm 6.0^{\mathrm{B}}$ & $52.8 \pm 5.4^{\mathrm{B}}$ & 0.135 \\
T2 & $52.7 \pm 5.9^{\mathrm{B}}$ & $59.6 \pm 8.7^{\mathrm{C}}$ & 0.001 \\
P & 0.001 & $<0.001$ & - \\
\hline
\end{tabular}

A generalized linear mixed model (with Bonferroni adjustment) was used to compare the ultrasonic values (PAAT) between the two groups at different observation time points. $\mathrm{P}>0.05$ when ${ }^{\mathrm{A}}$ compared to ${ }^{A}$, or ${ }^{B}$ compared to ${ }^{B}$. $P<0.05$ when ${ }^{A}$ compared to ${ }^{B}$, ${ }^{B}$ compared to ${ }^{C}$ or ${ }^{A}$ compared with ${ }^{C}$.

after birth $(\mathrm{P}=0.001)$.

\section{PDA incidence}

At baseline (T0), all neonates showed PDA, and no intergroup difference was found in relation to PDA diameter $(1.9 \pm 0.3$ vs. $1.9 \pm 0.4 \mathrm{~mm} ; \mathrm{P}=0.604)$. The transductal blood flow direction was exclusively left to right in all infants, and there was no significant difference in shunting pressure $(10.6 \pm 3.4$ vs. $12.3 \pm 3.5 \mathrm{mmHg} ; \mathrm{P}=0.065)$. At T1, PDA was observed in $62.5 \%$ of infants in the PS group, and $40 \%$ of infants in the control group $(\mathrm{P}=0.126)$. The PDA left-to-right shunt pressure was $11.2 \pm 3.3 \mathrm{vs}$. $13.2 \pm 3.8 \mathrm{mmHg}(\mathrm{P}=0.120)$ for the $\mathrm{PS}$ and control groups, respectively. There was no significant intergroup difference in relation to diameter $(1.97 \pm 0.29$ vs. $1.84 \pm 0.33 \mathrm{~mm}$; $\mathrm{P}=0.277)$. At 36 weeks PMA, PDA was only found in 1 infant in the PS group, and was not found in any infants in the control group.

\section{Discussion}

RDS is still the most common pulmonary disease in premature infants in the early postnatal period (1). The European Guidelines for the Management of Respiratory Distress Syndrome were drafted by a panel of expert neonatologists, and recommend the initiation of CPAP from birth, with early selective PS administration, for premature infants who show signs of RDS. The overall aim is to avoid MV where possible (or reduce its duration), improve neonatal survival rate, and minimize complications $(4,5)$. It is well known that the need for PS treatment can be used as a marker to judge the severity of RDS. The comprehensive management of RDS during the perinatal period enabled us
Table 3 The PAAT/RVET (ms/ms) values of the two groups

\begin{tabular}{lccc}
\hline Time & PS group & Control group & $P$ \\
\hline T0 & $0.29 \pm 0.03^{\mathrm{A}}$ & $0.28 \pm 0.04^{\mathrm{A}}$ & 0.090 \\
T1 & $0.29 \pm 0.02^{\mathrm{A}}$ & $0.31 \pm 0.03^{\mathrm{B}}$ & 0.033 \\
T2 & $0.30 \pm 0.03^{\mathrm{A}}$ & $0.33 \pm 0.03^{\mathrm{C}}$ & 0.001 \\
P & 0.117 & $<0.001$ & - \\
\hline
\end{tabular}

A generalized linear mixed model (with Bonferroni adjustment) was used to compare the ultrasonic values (PAAT/RVET) between the two groups at different observation time points. $\mathrm{P}>0.05$ when ${ }^{A}$ compared to ${ }^{A}$, or ${ }^{B}$ compared to ${ }^{B}$. $\mathrm{P}<0.05$ when ${ }^{A}$ compared to ${ }^{B},{ }^{B}$ compared to ${ }^{C}$ or $^{A}$ compared with ${ }^{C}$.

to avoid invasive ventilation in the early postnatal period for the vast majority of preterm infants with a GA $>26$ weeks at our center. To date, RDS research has mainly focused on methods of PS administration (e.g., traditional endotracheal intubation, Less Invasive Surfactant Administration or Minimally Invasive Surfactant Therapy), and the effects of different non-invasive support on future complications, such as BPD (3-5). As preterm birth and pulmonary disease can also disrupt the maturational development of pulmonary circulation, a greater understanding of preterm infants with presumed elevated pulmonary vascular resistance from pulmonary disease and difficulty with transition is also needed (7-11). Thus, based mainly on the above situation, a more homogeneous population (GA 26-31 weeks) was selected for this study. The groping criteria of whether PS treatment was needed was used to study the hemodynamic changes between the two groups of premature infants after birth.

The main findings of our research are as follows: (I) the preterm infants in the PS group had more severe clinical conditions, required longer hospital stays, had a higher incidence of complications, especially respiratory problems, and had a higher rate of BPD; (II) no significant intergroup difference was detected in terms of pressure difference in TR at the 3 time points; (III) at 36 weeks PMA, infants in the PS group had a significantly lower PAAT and PAAT/ RVET than the control group; and (IV) for the PS group, no significant increases in PAAT/RVET were detected at the three different time points, but both PAAT and PAAT/ RVET increased significantly with time after birth in the control group.

In the early stages of PVD in preterm infants, pulmonary artery compliance decreases while pulmonary vascular resistance increases. This may cause $\mathrm{PH}$ and lung disease to 
worsen when combined with RDS (7-11). Thus, conveniently, accurately, and sensitively estimating pulmonary vascular lesions among premature infants with RDS is of great significance in evaluating infant development and therapeutic effects. In relation to diagnosis and treatment, in addition to the clinical symptoms and physical examination, the dynamic monitoring of bedside echocardiography can be useful. Traditional echocardiographic criteria for diagnosing $\mathrm{PH}$ include a systolic PAP $>40 \mathrm{mmHg}$ (estimated based on the pressure difference in TR), a $\mathrm{PAP} /$ systemic systolic pressure $>0.5$, a cardiac shunt with bidirectional or right-to-left flow, and any degree of left-side septal flattening (23). However, in clinical practice, once these above-mentioned diagnostic criteria have been identified, the pulmonary lesions are often already serious. Thus, the traditional $\mathrm{PH}$-focused ultrasound diagnosis method provides delayed indications of the condition.

Our study found that TR was not available in all children (the detection rate on the first day after birth was $56 \%$ ). This is consistent with previous findings that have reported an inability to obtain a satisfactory TR Doppler signal in approximately $40 \%$ of patients (23). In addition, some studies involving cardiac catheterization have revealed that echocardiography-obtained pressure differences in TR may have a poor correlation with PAP (19). As in our study, none of the infants showed a right-to-left shunt in PDA, no significant intergroup difference was observed in relation to pressure differences in TR at the 3 time points, and no degree of left-side septal flattening was identified. As no significant intergroup difference in PAP was obtained using traditional methods, it was generally believed that there was no intergroup difference in terms of pulmonary lesion severity, but this may not be the case.

The early stages of PVD may cause a small increase in PAP in preterm infants (8). Thus, when conducting a conventional analysis of PAP alone (as discussed above), it is difficult to accurately determine the severity of PVD in a timely manner. Previous studies have found that other echocardiographic parameters are sensitive to increased pulmonary vascular resistance or pressure that occur before a diagnosis of $\mathrm{PH}$ can be made using conventional echocardiography $(20,21,24)$. In this study, we adopted the parameter of PAAT, recommended by Levy and Koestenberger [2016, 2017], which sensitively reflects PVD (21,25). Experts at the European Organizing Committee for Children's Pulmonary and Vascular Diseases 2014 recognized this parameter as an IIb indicator for the assessment of $\mathrm{PH}$ (26). Previous investigations have reported normal reference values for different ages. Specifically, research has shown that the average PAAT for normal infants in the first month is $80 \pm 13 \mathrm{~ms}$ (female) and $78 \pm 13 \mathrm{~ms}$ (male), and this increases with age (25). A recent study on premature infants at different GAs showed that among healthy premature infants, PAAT was approximately $44 \mathrm{~ms}$ on the first day after birth, $47 \mathrm{~ms}$ on the second day, and 57 ms at 36 weeks PMA. The overall PAAT trend continues to increase over time $(20,25,26)$. In our study, the mean PAAT values at T0 and T1 were slightly higher than those reported in the above studies; however, this may be due to the fact that the average GA of our study infants was greater than that of the other studies' patients.

It is well known that, as GA increases, lung development becomes more mature and right ventricular afterload is relatively reduced (20). However, at 36 weeks PMA, PAAT in the control group was close to that of healthy preterm infants while that of the PS group was significantly lower. Similar to clinical outcomes, preterm infants with RDS who needed PS treatment had more severe clinical symptoms, especially respiratory problems. We found that even with PS treatment, a late respiratory disease was still more likely to develop. Further, while the PAAT of the preterm infants in the control group increased gradually after birth, the level was still much lower than the results reported by previous studies on term infants $(25,26)$. Thus, it is our view that preterm birth-associated cardiopulmonary conditions may affect the maturational patterns of PAAT, and RDS exacerbates this effect. Due to the well-established inverse relationship between PAAT and HR (27), we simultaneously measured RVET to account for potential effects of HR variability, as the use of PAAT/RVET negates the need to correct for HR. We found that PAAT/RVET did not increase after PS treatment, and speculated that while PS treatment improved early postnatal dyspnea symptoms and reduced respiratory support requirements, it was not helpful in preliminary measurements of PAAT. Notably, previous studies have found that PAAT/RVET is inversely correlated with mean PAP (27), which may mean that the rapid decline in pulmonary vascular resistance in the first 1-2 days after birth is delayed for RDS preterm infants, especially those who need PS treatment (25).

Multiple studies have shown that PAAT is inversely correlated with the severity of $\mathrm{PH}$ and can be used to estimate PAP $(22,23)$. As PAAT does not rely on the presence of an anatomic defect or TR, it can be used to assess PAP in the vast majority of patients $(22,23)$. A recent study suggested that PAAT can be used as a complementary 
parameter to assess physiologic and pathologic changes in pulmonary hemodynamics in neonates (20). In our study's control group, PAAT continued to increase across the 3 different time points, which is generally consistent with the trend reported in previous studies of healthy premature infants whereby PAAT increased over time in the neonatal period $(26,28)$. Our results showed that, as premature infants grow and diseases are treated, pulmonary vascular compliance increases significantly, and PAP continues to decline. However, at 36 weeks PMA, PAAT in the PS group was significantly lower than that in the control group, and the incidence of BPD in the PS group was much higher, indicating the continuous progression of pulmonary vascular lesions.

Recent studies have shown that preterm infants with BPD have lower PAAT at 32 and 36 weeks PMA than those with no BPD (26), which indicates that PAAT is sensitive to pulmonary vascular conditions both in the early and late postnatal stages. Thus, it may be used as a reliable non-invasive screening measure for evaluating pulmonary hemodynamics in premature infants with RDS and late respiratory disease. Several studies have also shown that children with BPD still have respiratory problems 1 year or more after birth, and that their PAP is consistently higher than that of normal children of the same age $(7,11,13,25,26)$. Thus, premature infants with RDS, especially those requiring PS treatment, are more likely to develop PVD in the future. In addition to early interventions with appropriate PS treatment and respiratory support and other therapeutic measures, long-term continuous monitoring is required, which may improve their respiratory prognoses.

It is often difficult to clinically distinguish significant differences in lung lesions between and within RDS individuals using chest radiographs alone. With the recent rapid development of pulmonary ultrasound technology, some experts have used lung ultrasounds to examine premature infants with RDS. In these investigations, lung lesions were found to be less severe in infants who did not require PS treatment. Such infants showed signs of smallscale focal consolidation and mild alveolar interstitial syndrome, while those who needed PS treatment showed signs of extensive lung consolidation or "white lung" and more serious lung lesions (29,30). A pulmonary ultrasound score may be capable of predicting the need for PS treatment in premature infants with a GA of $<34$ weeks $(30,31)$.

In addition to the echocardiographic prediction PAAT parameters used in our research, in recent studies, Ehrmann et al. [2018] noted that the eccentricity index may be a useful echocardiographic measurement for characterizing right ventricle mechanics in patients with BPD at 36 weeks PMA $(32,33)$. The eccentric index is mostly used in the research of BPD, but rarely in studies on early pulmonary lesions in premature infants. As a potentially useful metric, the eccentric index may be used as a joint research indicator for the further study of early pulmonary lesions in premature infants, including those with RDS. In the near future, with the combination of pulmonary ultrasound and echocardiography and other imaging methods, it may be possible to better clarify the pulmonary hemodynamics of preterm infants with RDS, and predict which preterm infants are likely to develop severe PVD via early warnings and quantifying the severity degree of BPD, which would allow targeted therapy with oxygen, pulmonary vasodilators, or different respiratory support strategies to be adopted.

Most premature infants have PDA in the early postnatal period, and PAP can be precisely measured with a PDA (assuming that a reliable Doppler measurement can be obtained). PAP is the systemic pressure minus the PDA gradient. However, in premature infants, especially those in the lower systemic blood pressure range, blood pressure (as measured by a cuff) does not correlate well with invasive measurements $(34,35)$. Thus, a PAP measurement based on a PDA gradient without a simultaneous invasive BP should be interpreted with caution. In these cases, PAAT would be more reliable, as knowledge of the $\mathrm{BP}$ is not required to estimate it. Further, the arterial ducts of most newborns are functionally closed within 96 hours after birth and cannot be dynamically used as an indicator to monitor PAP (36).

Most studies consider PAAT to be a convenient, accurate, and sensitive ultrasound parameter for evaluating pulmonary hemodynamics; however, some studies have suggested that as most preterm infants also have concurrent PDA, the turbulence of the PDA left-to-right shunt can affect the flow direction and velocity in pulmonary artery lumen, and thus this parameter is not suitable for preterm infants (27). In our study, which comprised a carefully selected and homogeneous population, the ductus arteriosus was closed in almost all preterm infants at PMA 36 weeks. Early after birth at T0 and T1, the most PDA measurements did not have hemodynamic significance, and there was no increase in heart rate, differential pulse pressure, or the ratio of the left atrium to aorta. Thus, it is not symptomatic PDA that needs to be treated (27). Further, in clinical practice, PAAT was measured at the pulmonary valve orifice. A PDA left-to-right shunt is usually attached to the left lateral wall of the main 
pulmonary artery, and a PDA shunt in most premature infants does not reach the level of the pulmonary valve orifice; thus, PAAT can be accurately measured by carefully avoiding the shunt beam.

This study had some limitations. First, it did not consider more testing time points, such as 72 hours after birth or 40 weeks PMA, or have a long-term follow-up period after discharge. Such time points would have enabled us to evaluate the dynamic trend of PAAT more comprehensively. Second, this study did not conduct concurrent crosssectional research on healthy preterm neonates who were not receiving respiratory support. Finally, we did not perform simultaneous pulmonary ultrasound examinations for all preterm neonates, which may have provided considerable information regarding the correlation between pulmonary lesions and pulmonary PAAT.

In conclusion, premature infants with RDS, especially those requiring PS treatment, are more likely to develop PVD in the future; thus, it is very important to monitor their pulmonary hemodynamics. As a convenient measurement parameter, PAAT appears to be a reliable non-invasive screening measure for evaluating pulmonary hemodynamics in premature infants with RDS and late PVD.

\section{Acknowledgments}

Funding: This research was supported by the Projects of Medical and Health Technology Development Program in Shandong Province (2016WS0483).

\section{Footnote}

Reporting Checklist: The authors have completed the STROBE reporting checklist. Available at https://dx.doi. org/10.21037/tp-21-341

Data Sharing Statement: Available at https://dx.doi. org/10.21037/tp-21-341

Conflicts of Interest: All authors have completed the ICMJE uniform disclosure form (available at https://dx.doi. org/10.21037/tp-21-341). The authors have no conflicts of interest to declare.

Ethical Statement: The authors are accountable for all aspects of the work in ensuring that questions related to the accuracy or integrity of any part of the work are appropriately investigated and resolved. All procedures performed in this study involving human participants were in accordance with the Declaration of Helsinki (as revised in 2013). This study was approved by the Ethics Committee of the First Affiliated Hospital of Shandong First Medical University, and informed consent was obtained from the children's parents or legal guardians.

Open Access Statement: This is an Open Access article distributed in accordance with the Creative Commons Attribution-NonCommercial-NoDerivs 4.0 International License (CC BY-NC-ND 4.0), which permits the noncommercial replication and distribution of the article with the strict proviso that no changes or edits are made and the original work is properly cited (including links to both the formal publication through the relevant DOI and the license). See: https://creativecommons.org/licenses/by-nc-nd/4.0/.

\section{References}

1. Bae CW, Kim CY, Chung SH, et al. History of Pulmonary Surfactant Replacement Therapy for Neonatal Respiratory Distress Syndrome in Korea. J Korean Med Sci 2019;34:e175.

2. Dyer J. Neonatal Respiratory Distress Syndrome: Tackling A Worldwide Problem. P T 2019;44:12-4.

3. McPherson C, Wambach JA. Prevention and Treatment of Respiratory Distress Syndrome in Preterm Neonates. Neonatal Netw 2018;37:169-77.

4. Sweet DG, Carnielli V, Greisen G, et al. European Consensus Guidelines on the Management of Respiratory Distress Syndrome - 2019 Update. Neonatology 2019;115:432-50.

5. Sweet DG, Carnielli V, Greisen G, et al. European Consensus Guidelines on the Management of Respiratory Distress Syndrome - 2016 Update. Neonatology 2017;111:107-25.

6. Glass HC, Costarino AT, Stayer SA, et al. Outcomes for extremely premature infants. Anesth Analg 2015;120:1337-51.

7. Mourani PM, Abman SH. Pulmonary Hypertension and Vascular Abnormalities in Bronchopulmonary Dysplasia. Clin Perinatol 2015;42:839-55.

8. Lau EM, Manes A, Celermajer DS, et al. Early detection of pulmonary vascular disease in pulmonary arterial hypertension: time to move forward. Eur Heart J 2011;32:2489-98.

9. Copetti R, Cattarossi L, Macagno F, et al. Lung ultrasound in respiratory distress syndrome: a useful tool for early 
diagnosis. Neonatology 2008;94:52-9.

10. Vitali F, Galletti S, Aceti A, et al. Pilot observational study on haemodynamic changes after surfactant administration in preterm newborns with respiratory distress syndrome. Ital J Pediatr 2014;40:26.

11. Mourani PM, Mandell EW, Meier M, et al. Early Pulmonary Vascular Disease in Preterm Infants Is Associated with Late Respiratory Outcomes in Childhood. Am J Respir Crit Care Med 2019;199:1020-7.

12. Mirza H, Ziegler J, Ford S, et al. Pulmonary hypertension in preterm infants: prevalence and association with bronchopulmonary dysplasia. J Pediatr 2014;165:909-14.e1.

13. Mourani PM, Sontag MK, Younoszai A, et al. Early pulmonary vascular disease in preterm infants at risk for bronchopulmonary dysplasia. Am J Respir Crit Care Med 2015;191:87-95.

14. O'Byrne ML, Kennedy KF, Kanter JP, et al. Risk Factors for Major Early Adverse Events Related to Cardiac Catheterization in Children and Young Adults With Pulmonary Hypertension: An Analysis of Data From the IMPACT (Improving Adult and Congenital Treatment) Registry. J Am Heart Assoc 2018;7:008142.

15. Lovrenski J, Sorantin E, Stojanović S, et al. Evaluation of Surfactant Replacement Therapy Effects--A New Potential Role of Lung Ultrasound. Srp Arh Celok Lek 2015;143:669-75.

16. Abman SH, Hansmann G, Archer SL, et al. Pediatric Pulmonary Hypertension: Guidelines From the American Heart Association and American Thoracic Society. Circulation 2015;132:2037-99.

17. Jobe AH, Bancalari E. Bronchopulmonary dysplasia. Am J Respir Crit Care Med 2001;163:1723-9.

18. Higgins RD, Jobe AH, Koso-Thomas M, et al. Bronchopulmonary Dysplasia: Executive Summary of a Workshop. J Pediatr 2018;197:300-8.

19. McGoon M, Gutterman D, Steen V, et al. Screening, early detection, and diagnosis of pulmonary arterial hypertension: ACCP evidence-based clinical practice guidelines. Chest 2004;126:14S-34S.

20. Patel MD, Breatnach CR, James AT, et al. Echocardiographic Assessment of Right Ventricular Afterload in Preterm Infants: Maturational Patterns of Pulmonary Artery Acceleration Time Over the First Year of Age and Implications for Pulmonary Hypertension. J Am Soc Echocardiogr 2019;32:884-894.e4.

21. Levy PT, Patel MD, Groh G, et al. Pulmonary Artery Acceleration Time Provides a Reliable Estimate of
Invasive Pulmonary Hemodynamics in Children. J Am Soc Echocardiogr 2016;29:1056-65.

22. Cowie B, Kluger R, Rex S, et al. The relationship between pulmonary artery acceleration time and mean pulmonary artery pressure in patients undergoing cardiac surgery: An observational study. Eur J Anaesthesiol 2016;33:28-33.

23. Yared K, Noseworthy P, Weyman AE, et al. Pulmonary artery acceleration time provides an accurate estimate of systolic pulmonary arterial pressure during transthoracic echocardiography. J Am Soc Echocardiogr 2011;24:687-92.

24. Habash S, Laser KT, Moosmann J, et al. Normal values of the pulmonary artery acceleration time (PAAT) and the right ventricular ejection time (RVET) in children and adolescents and the impact of the PAAT/RVETindex in the assessment of pulmonary hypertension. Int J Cardiovasc Imaging 2019;35:295-306.

25. Koestenberger M, Grangl G, Avian A, et al. Normal Reference Values and z Scores of the Pulmonary Artery Acceleration Time in Children and Its Importance for the Assessment of Pulmonary Hypertension. Circ Cardiovasc Imaging 2017;10:e005336.

26. Koestenberger M, Apitz C, Abdul-Khaliq H, et al. Transthoracic echocardiography for the evaluation of children and adolescents with suspected or confirmed pulmonary hypertension. Expert consensus statement on the diagnosis and treatment of paediatric pulmonary hypertension. The European Paediatric Pulmonary Vascular Disease Network, endorsed by ISHLT and D6PK. Heart 2016;102 Suppl 2:ii14-22.

27. Gaulton JS, Mercer-Rosa LM, Glatz AC, et al. Relationship between pulmonary artery acceleration time and pulmonary artery pressures in infants. Echocardiography 2019;36:1524-31.

28. Evans NJ, Archer LN. Postnatal circulatory adaptation in healthy term and preterm neonates. Arch Dis Child 1990;65:24-6.

29. Lovrenski J. Lung ultrasonography of pulmonary complications in preterm infants with respiratory distress syndrome. Ups J Med Sci 2012;117:10-7.

30. Piette E, Daoust R, Denault A. Basic concepts in the use of thoracic and lung ultrasound. Curr Opin Anaesthesiol 2013;26:20-30.

31. Brat R, Yousef N, Klifa R, et al. Lung Ultrasonography Score to Evaluate Oxygenation and Surfactant Need in Neonates Treated With Continuous Positive Airway Pressure. JAMA Pediatr 2015;169:e151797. 
32. Ehrmann DE, Mourani PM, Abman SH, et al. Echocardiographic Measurements of Right Ventricular Mechanics in Infants with Bronchopulmonary Dysplasia at 36 Weeks Postmenstrual Age. J Pediatr 2018;203:210-217.e1.

33. McCrary AW, Malowitz JR, Hornick CP, et al.

Differences in Eccentricity Index and Systolic-Diastolic Ratio in Extremely Low-Birth-Weight Infants with Bronchopulmonary Dysplasia at Risk of Pulmonary Hypertension. Am J Perinatol 2016;33:57-62.

34. Takci S, Yigit S, Korkmaz A, et al. Comparison between oscillometric and invasive blood pressure measurements

Cite this article as: Wang L, Zhang F, Li J, Liu Z, Kou Y, Song Y, Xu H, Wang H, Wang Y. Using pulmonary artery acceleration time to evaluate pulmonary hemodynamic changes on preterm infants with respiratory distress syndrome. Transl Pediatr 2021;10(9):2287-2297. doi: 10.21037/tp-21-341 in critically ill premature infants. Acta Paediatr 2012;101:132-5.

35. Cambiaso-Daniel J, Rontoyanni VG, Foncerrada G, et al. Correlation between invasive and noninvasive blood pressure measurements in severely burned children. Burns 2018;44:1787-91.

36. Mohammad Nijres B, Bokowski J, Mubayed L, et al. Utility of Pulmonary Artery Acceleration Time to Estimate Systolic Pulmonary Artery Pressure in Neonates and Young Infants. Pediatr Cardiol 2020;41:265-71. 